\title{
Development of focal plane detectors for the Nuclear Spectroscopic Telescope Array (NuSTAR) mission
}

Vikram R. Rana, Walter R. Cook, Fiona A. Harrison, Peter H. Mao, Hiromasa Miyasaka

Vikram R. Rana, Walter R. Cook, Fiona A. Harrison, Peter H. Mao, Hiromasa Miyasaka, "Development of focal plane detectors for the Nuclear

Spectroscopic Telescope Array (NuSTAR) mission," Proc. SPIE 7435, UV, XRay, and Gamma-Ray Space Instrumentation for Astronomy XVI, 743503 (3 September 2009); doi: 10.1117/12.825418

SPIE Event: SPIE Optical Engineering + Applications, 2009, San Diego, California, United States 


\title{
Development of Focal Plane Detectors for the Nuclear Spectroscopic Telescope Array (NuSTAR) Mission
}

\author{
Vikram R. Rana ${ }^{\mathrm{a}}$, Walter R. Cook III', Fiona A. Harrison ${ }^{\mathrm{a}}$, Peter H. Mao ${ }^{\mathrm{a}}$, Hiromasa Miyasaka ${ }^{\mathrm{a}}$ \\ ${ }^{\text {a }}$ Space Radiation Laboratory, California Institute of Technology, Pasadena, CA 91125
}

\begin{abstract}
The Nuclear Spectroscopic Telescope Array (NuSTAR), scheduled for launch in 2011, is a NASA Small Explorer mission that will improve the current sensitivity for detection of faint astrophysical sources in the $6-80 \mathrm{keV}$ band by two orders of magnitude. NuSTAR achieves high sensitivity by utilizing a hard X-ray focusing system. We have developed Cadmium Zinc Telluride (CdZnTe) pixel detectors optimized for good energy for the NuSTAR focal plane. Each of NuSTAR's two focal planes is comprised of hybrid detectors that consist of a CdZnTe pixel sensor with the anode contacts directly attached to corresponding readout circuits integrated on a custom low-noise VLSI chip. Each hybrid is $20.5 \times 20.5 \times 2.0 \mathrm{~mm}$ in size with the anode divided into $32 \times 32$ array of pixels at $0.6048 \mathrm{~mm}$. In this paper we describe the hybrid sensor architecture, and present preliminary results from the characterization of detectors fabricated for the NuSTAR focal plane Engineering Test Unit (ETU). We achieve excellent electronic readout noise with an average of $250 \mathrm{eV} \mathrm{FWHM}$, and energy resolution between 0.9 and $1.6 \mathrm{keV}$ FWHM at $86.5 \mathrm{keV}$, depending on position in the sensor and improving at lower energies. In order to achieve the best spectral resolution we need to make pixeldependent corrections for events with charge split among multiple pixels, and in addition we make spectral corrections based on depth of the gamma-ray interaction.
\end{abstract}

Keywords: Hard X-ray astrophysics, pixilated CdZnTe detectors, electronic noise and spectral performance, NuSTAR

\section{INTRODUCTION}

Cadmium Zinc Telluride (CdZnTe) is being widely developed for use in space-based astrophysical hard X-ray and gamma-ray applications. It offers the ability to implement compact, low-mass solid state detector arrays with good position resolution and spectral resolution significantly improved over traditional alkali halide scintillators ${ }^{1,2}$. CdZnTe can also be operated with relatively low leakage currents with modest cooling (temperatures on order $0{ }^{\circ} \mathrm{C}$ ).

Our group at Caltech has developed a hard X-ray pixel detector based on CdZnTe material for use in the focal planes of astrophysical imaging telescopes. A first-generation version of the detector was flown on the High Energy Focusing Telescope $(H E F T)^{3}$ balloon experiment, and we have significantly improved the design for the Nuclear Spectroscopic Telescope Array (NuSTAR) NASA Small Explorer mission, scheduled to launch in August 2011. NuSTAR will be the first space mission to employ hard X-ray focusing telescope to image the sky in $6-80 \mathrm{keV}$ energy band, providing a combination of high sensitivity and good energy resolution. NuSTAR has two co-aligned telescopes each consist of depth-graded multilayer optics focusing onto an actively-shielded CdZnTe focal plane.

In this paper we describe the development of the CdZnTe pixel detectors. We present the performance requirements for the NuSTAR focal plane, and describe the detector architecture, which has been optimized for this application.

\section{NUSTAR FOCAL PLANE REQUIREMENTS}

NuSTAR consists of to co-aligned hard X-ray telescopes. Each telescope is a conical approximation to a Wolter I design with a 10 meter focal length,5. The multilayer-coated optics will have angular resolution of 43 arcseconds (half power

UV, X-Ray, and Gamma-Ray Space Instrumentation for Astronomy XVI,

edited by Oswald H. Siegmund, Proc. of SPIE Vol. 7435, 743503 · (c) 2009 SPIE

CCC code: $0277-786 X / 09 / \$ 18 \cdot$ doi: $10.1117 / 12.825418$ 
diameter), and energy dependent field of view that is 12 arcminutes (FWHM) at $6 \mathrm{keV}$ and 7 arcminutes at $60 \mathrm{keV}$. The mission is designed for faint source detection and mapping of diffuse features, so that high count rate handling is not required. NuSTAR's key science objectives include mapping of ${ }^{44} \mathrm{Ti}$ lines at 68 and $78 \mathrm{keV}$ in young supernovae, requiring good spectral resolution for both detection sensitivity and accurate line centroiding. The requirements on the focal plane system that result from these considerations are listed in Table 1.

Table 1: Key requirements for the NuSTAR focal plane system.

\begin{tabular}{|l|c|}
\hline Item & Requirement \\
\hline Energy range & $6-80 \mathrm{keV}$ \\
\hline Single pixel energy threshold & $<4 \mathrm{keV}$ \\
\hline $\begin{array}{l}\text { Spectral resolution } \\
\text { (Each 10 x 10 pixel region) }\end{array}$ & $<1.6 \mathrm{keV} \mathrm{FWHM}(6-80 \mathrm{keV})$ \\
\hline Operating temperature & $0-10{ }^{\circ} \mathrm{C}$ \\
\hline Count rate handling & $>100$ events $/ \mathrm{s} / \mathrm{telescope}$ \\
\hline Readout dead time & $<3$ milliseconds/event \\
\hline Temporal resolution & $<1 \mathrm{msec}$ \\
\hline
\end{tabular}

\section{DETECTOR CONFIGURATION}

Each of the two NuSTAR focal plane module (FPM) consists of four CdZnTe hybrid detectors arranged in 2x2 array. Each hybrid consists of a CdZnTe sensor with a segmented anode with each pixel attached to a readout circuit on a custom designed low-noise ASIC. Both the readout circuits and the anode contacts are placed on a 32 x 32 grid with 605 micron pitch. The hybrid size is chosen as the maximum that can be accommodated by the ASIC foundry, and also a size compatible with selecting high-quality single-crystal CdZnTe sensors. Each ASIC contains an on-chip analog-todigital converter (ADC), and an external microprocessor supports the readout.

The CdZnTe crystals were purchased from eV Products. Each detector is about $2 \times 2 \times 0.2 \mathrm{~cm}$ in size. The cathode is a monolithic platinum contact and the anode plane is patterned into a $32 \times 32$ pixel array with each pixel having pitch of about $605 \mu \mathrm{m}$ forming 1024 pixels for each detector and about $50 \mu \mathrm{m}$ gap between pixel contacts. Figure 1 shows the detector anode pattern for CdZnTe with a magnified view of a few corner pixels, showing measured distances. The picture on the right side shows the NuSTAR focal plane engineering test unit. Each hybrid is mounted on a ceramic carrier board, and each hybrid/carrier board assembly can be independently replaced.
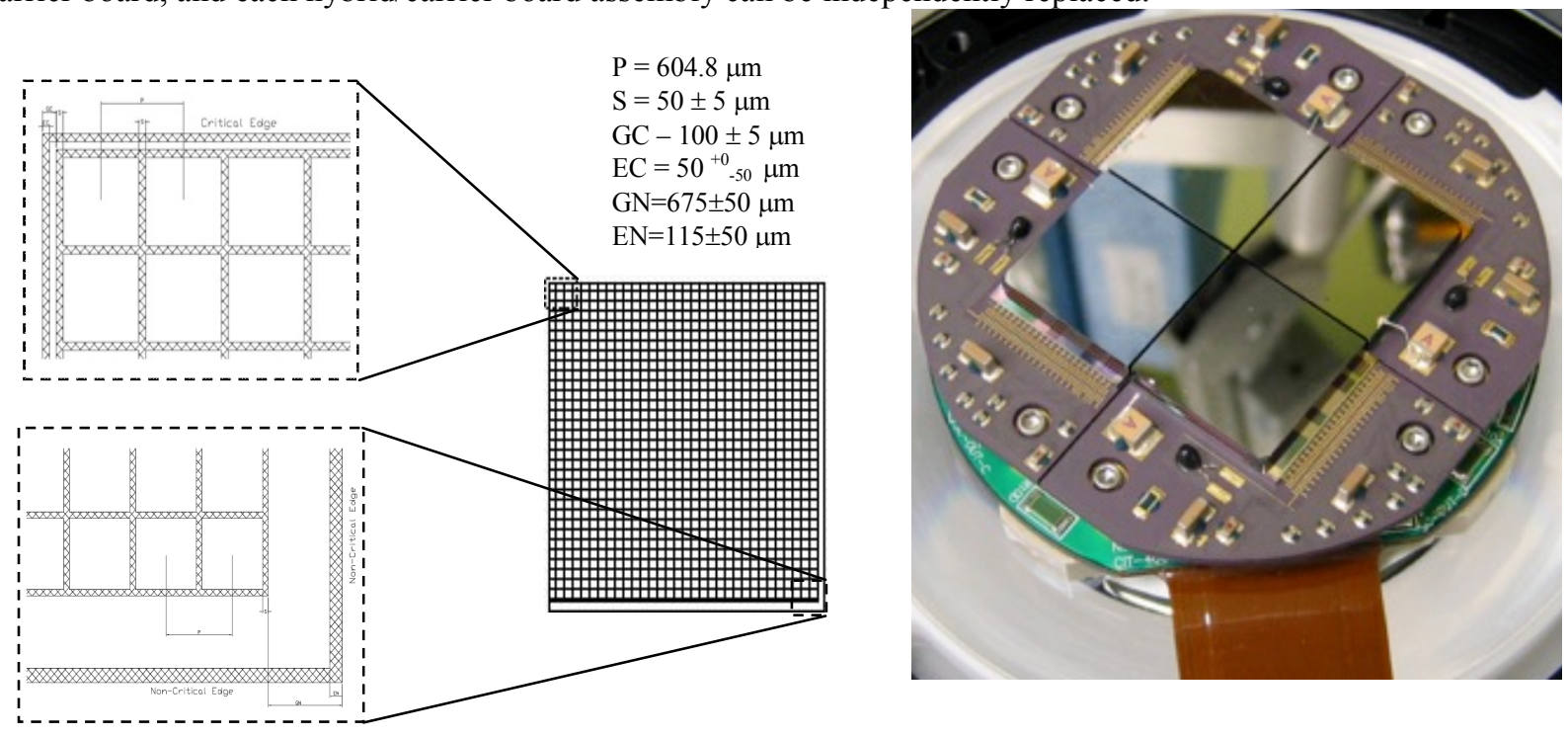
Figure 1. Left: NuSTAR CZT detector anode pattern showing a few pixel geometry of the two diagonal corners of entire $32 \times 32$ pixels array. Right: Four hybrids assembled together forming a focal plane module of the NuSTAR.

The NuSTAR custom low-noise ASIC readout was developed at Caltech with the design optimized to achieve the best possible energy resolution while meeting the image sampling constraints. Each pixel has its own preamplifier, shaping amplifier, discriminator, sample and hold circuitry and test pulsar. The ASIC was originally developed for the HEFT balloon experiment and has been upgraded to use for NuSTAR satellite mission. The upgraded version has two readout modes: a Normal Mode and a Charge Pump (CP) Mode. The normal mode has the capacity to handle high leakage current up to $10 \mathrm{nA}$ per pixel, making it easy to operate the hybrids at room temperature - it is therefore very useful for routine laboratory testing of hybrids without cooling, and for functional evaluation during instrument integration and environmental testing. On the other hand, CP mode has significantly lower electronic noise, but can handle leakage current only up to $300 \mathrm{pA}$ per pixel. It achieves the optimal spectral resolution, obtainable when the detector is moderately cooled to $\sim 0^{\circ} \mathrm{C}$. In addition, the ASIC has on-chip 12-bit Analog-to-Digital Converter (ADC). Finally, the ASIC is designed to trigger only on negative pulses, but it has sensitivity to both positive and negative polarities, so that both electron and hole signals can be detected, allowing us to measure the depth of interaction of each X-ray inside CZT detector.

The ASICs are directly bonded to the CdZnTe sensors using a conductive epoxy/gold stud interconnect. Gold studs are placed on the ASIC pads, conductive epoxy is screened on the detector, and the two are hybridized using a flip-chip process. The height of the gold stud interconnect is 50 micron, which is sufficient to minimize the contribution of the capacitance between the CdZnTe anode and ASIC to the total input capacitance, which is dominated by the contact-tocontact term.

\section{CHARACTERISTICS}

Six hybrids (CdZnTe + ASIC) have been fully fabricated and tested for their overall performance. We selected 4 hybrids and assembled them for the engineering model (EM) of the focal plane detector system. Here we report on some of the early results from these hybrids. For the flight units, we plan to hybridize more than $50 \mathrm{CZT}$ detectors to select the eight best for flight, four for the each of two NuSTAR focal plane modules. All the CdZnTe crystals have been purchased from eV Products. The CdZnTe sensors and ASICs undergo detailed screening procedure before and after hybridization to qualify as a potential flight hybrid.

Before hybridization $\mathrm{eV}$ Products does IR imaging to select good material from the CdZnTe wafer. The IR images are used to select regions without large Te precipitate densities or inclusions. The ASICs also undergo a detailed screening that includes an AC test, DC test, and noise measurement before hybridizing with a CdZnTe sensor. After CdZnTe and ASIC are bonded together, the hybrid undergoes a screening process including mechanical inspection, noise measurement, and leakage current measurements. The hybrids that pass through above steps undergo a temperature scan between $-15 \mathrm{C}$ to $+20 \mathrm{C}$ with $\gamma$-ray flood illumination from a radioactive source to determine the ideal operating temperature for each hybrid. Once the optimum operating temperature is known, we take long $\gamma$-ray flood illumination data to fully characterize the spectral properties and uniformity.

\subsection{Electronic noise}

The electronic noise of the system can be measured by artificially stimulating the detector to trigger, and measuring the distribution of resultant pulse heights with no signal present. Data used for this measurement were collected at $0 \mathrm{C}$ temperature without the high voltage bias. Figure 1 shows the electronic noise measurements for one of the $\mathrm{CdZnTe}$ hybrid. The left hand side of the figure shows a map representing electronic noise in each pixel in a $32 \times 32$ pixel configuration of the detector with corresponding color scale at the top. A histogram distribution of the noise measurements for all pixels is shown on the right hand side. The histogram readily shows that almost all pixels have 
electronic noise between $200 \mathrm{eV}$ to $400 \mathrm{eV}$ with peak at about $250 \mathrm{eV}$. This exceeds the requirement of about $800 \mathrm{eV}$ for the NuSTAR.
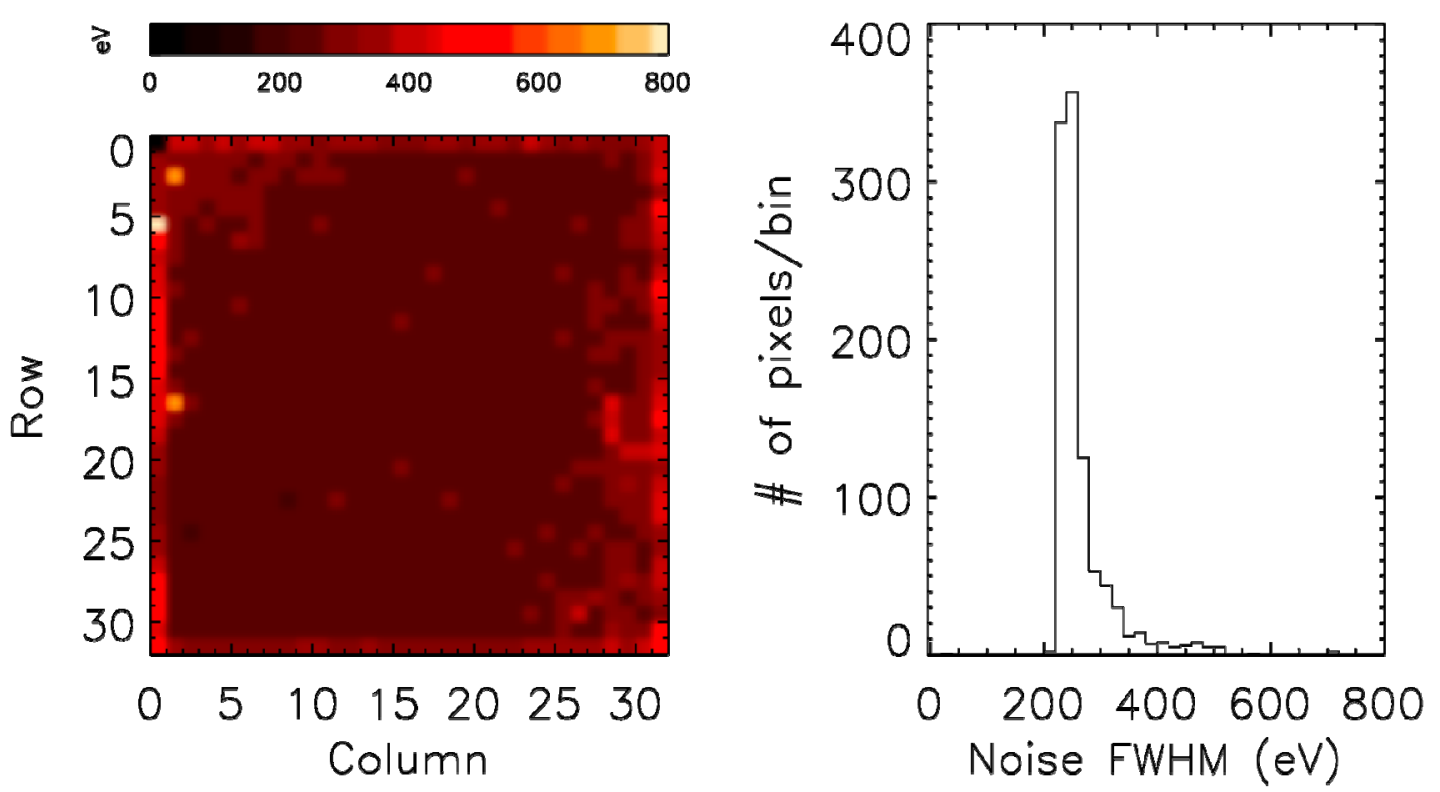

Fig. 2. Left: Map showing electronic noise for each pixel in the hybrid with corresponding color bar at the top. Right side shows the histogram distribution of the electronic noise. The data were taken at room temperature without high voltage bias.

\subsection{Counts Uniformity and Split Pixel Events}

To measure the count rate uniformity of these detectors, which is important for good imaging performance, we flood illuminate the detector with a non-collimated radioactive source, and measure event counts in each pixel. We used ${ }^{155} \mathrm{Eu}$ source with $100 \mu \mathrm{Ci}$ strength for this purpose. It provides prominent spectral lines at about $43 \mathrm{keV}, 86.5 \mathrm{keV}$ and 105 $\mathrm{keV}$. Figure 3 shows the counts map on the left panel and corresponding histogram in the right panel for two of our EM CZT detectors. These data were collected at $7 \mathrm{C}$ temperature and $-300 \mathrm{~V}$ bias voltages. Each dot in the map represents an event registered in that pixel. Events have been randomized over pixel area for better presentation. Several pixels were intentionally disabled due to high noise. The count distribution is significantly more uniform for the hybrid shown on the top compared to the bottom of the figure. In both cases, however, the distribution is broader than what would be expected statistically from the finite number of counts in each pixel. In the hybrid shown in the lower panel, some structural features are clearly visible in the central area. The counts histogram for the uniform detector is narrower and symmetric, whereas the distribution is broader with a long tail for the non-uniform detector. This non-uniformity over the detector area is apparently due to the existence of a transverse component of the electric field in the detector that changes effective size of pixels and thus their charge collection efficiency. To achieve the requisite image reconstruction for NuSTAR, we plan to calibrate each flight detector with a collimated $50-100$ micron diameter beam in order to map the locations of pixel centroids. 

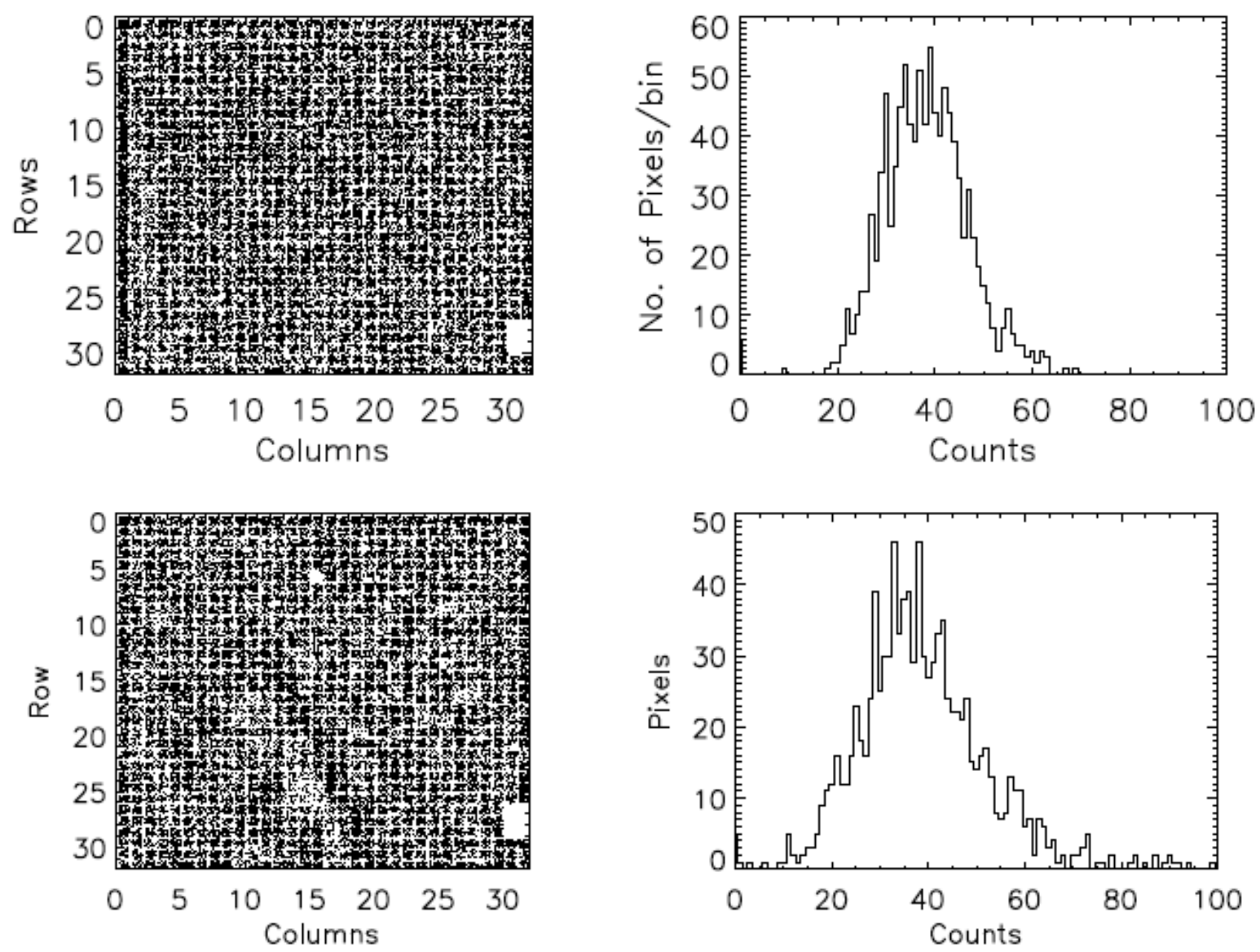

Figure 3. Left: Counts map for a CZT hybrid showing number of counts in each pixel. Each dot in the map represents an event in that pixel. Six pixels on lower right corner without any events were turned-off since they were found to be noisy. Right: Histogram showing corresponding distribution of counts. Data used were collected from $155 \mathrm{Eu}$ source at $7 \mathrm{C}$ with $-300 \mathrm{~V}$ bias voltages.

For detectors with small pixels, a significant fraction of events have charge split among multiple pixels. For the NuSTAR hybrids more that $40 \%$ of the total events show charge sharing. We have investigated the charge-sharing effect in detail for the NuSTAR CZT detectors and estimated the fractional ratio of events that deposited charge in 1,2, 3, 4, and more than 5 pixels. For this purpose we again used the same ${ }^{155} \mathrm{Eu}$ source data, as mentioned above. It was observed that about $53 \%$ of the total events have deposited entire charge into a single pixel whereas rest of the $47 \%$ of events deposit charge in $2,3,4$, and more than 5 pixels. Charge splitting between 2 or 3-pixels can happen when charge interaction takes place near the edge or corner of the pixel. On the other hand charge sharing between 5 and more pixel occurs when there is significant electron trapping, where the signal is generated due to charge being imaged on the pixels by the trapped electrons.

\subsection{Spectral Resolution}

In order to meet the science requirements for NuSTAR the CdZnTe detector must have energy resolution $\leq 1.6 \mathrm{keV}$ FWHM over the entire energy range for any 10x10 pixel region on the detector area. In order to characterize the spectral properties of NuSTAR CZT detectors, we used same flood illumination data from ${ }^{155} \mathrm{Eu}$ radioactive source as described in the previous section. The data were collected for sufficiently long time to accumulate enough counts in each pixel. The data clearly show pixel-to-pixel gain variations due to charge collection non-uniformities that dominate over the small mismatches in electronics gains. This pixel-to-pixel detector-induced gain variation broadens the spectral lines significantly if not corrected. In order to quantify this variation, we fit a Gaussian to most prominent $86.5 \mathrm{keV}$ line in 
the spectrum to determine the line centroid for each pixel. We find for a typical detector that the line position shows about a $10 \%$ of shift for all 1024 pixels.

In order to illustrate the spectral performance of the NuSTAR EM hybrids, we randomly selected five $3 \times 3$ pixel regions (four near corners and one at centre) over the detector area and plot spectra for each of these regions. For the sake of simplicity, we selected single pixel triggered events only and rejected all the events that show charge sharing between two or more pixels. Figure 5 shows these five spectra along with FWHM energy resolution for $86.5 \mathrm{keV}$ line. We have applied pixel-to-pixel gain correction to these spectra. It can be seen from the figure that this hybrid does show some variation in spectral performance over the detector area; in particular, the spectrum from the central region of the CZT detector has comparatively higher FWHM than the other region on the detector. This spectral variation is clearly due to inhomogeneities in the CdZnTe. In the spectra below we have not corrected for depth of interaction effects, which improves spectral resolution at high energies $(>50 \mathrm{keV})$.
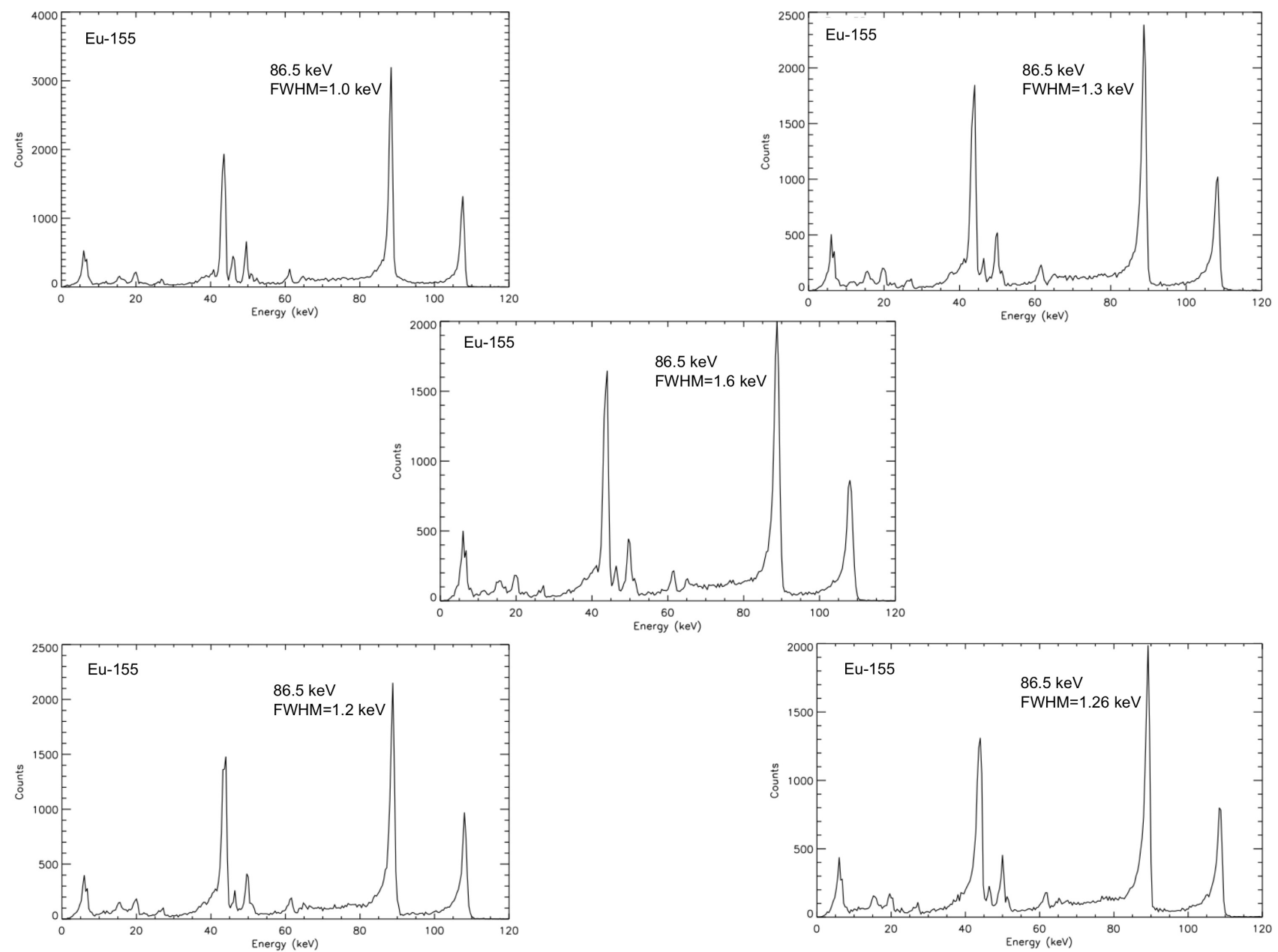

Figure 5: 155Eu spectra for the five $3 \times 3$ pixel area randomly selected over detector area (four from corner and one at centre). Data were taken at $280 \mathrm{~K}$ temperature and $-300 \mathrm{~V}$ high voltage. Events only from single-pixel triggered have been used. Spectra are corrected for the pixel-to-pixel detector gain variation.

To further study the charge sharing between pixels, we also generated ${ }^{155} \mathrm{Eu}$ spectrum including all kind of events, single pixel triggered plus two or more triggered pixels, for a $3 \times 3$ pixel region. A detailed study of charge sharing events suggest that we need to account for pixel dependent charge sharing patterns to fully correct the spectra and obtain the best possible energy resolution. The left panel in Figure 6 shows 13 such patterns for a 9-pixel readout accounting for up to 4 triggered pixels. The right panel shows a spectrum from all events including single pixel and charge sharing pixels. Generating such a large number of parameters for each detector is a very time consuming process and requires a lot of 
calibration data. For a 9-pixel readout there are total of 13 different event topologies that need to be taken into account for each pixel. To correct pattern dependent detector gain variations for every pixel would require a total of 13,312 parameters for each detector. We are in the process of determining the minimum set necessary for optimal spectral resolution.
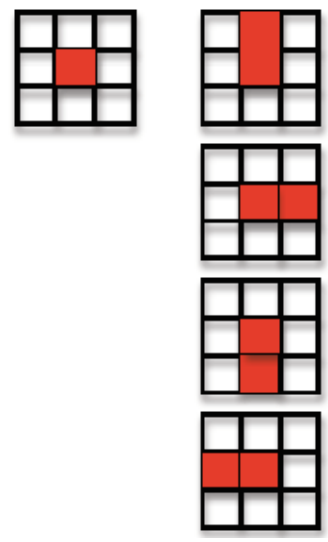
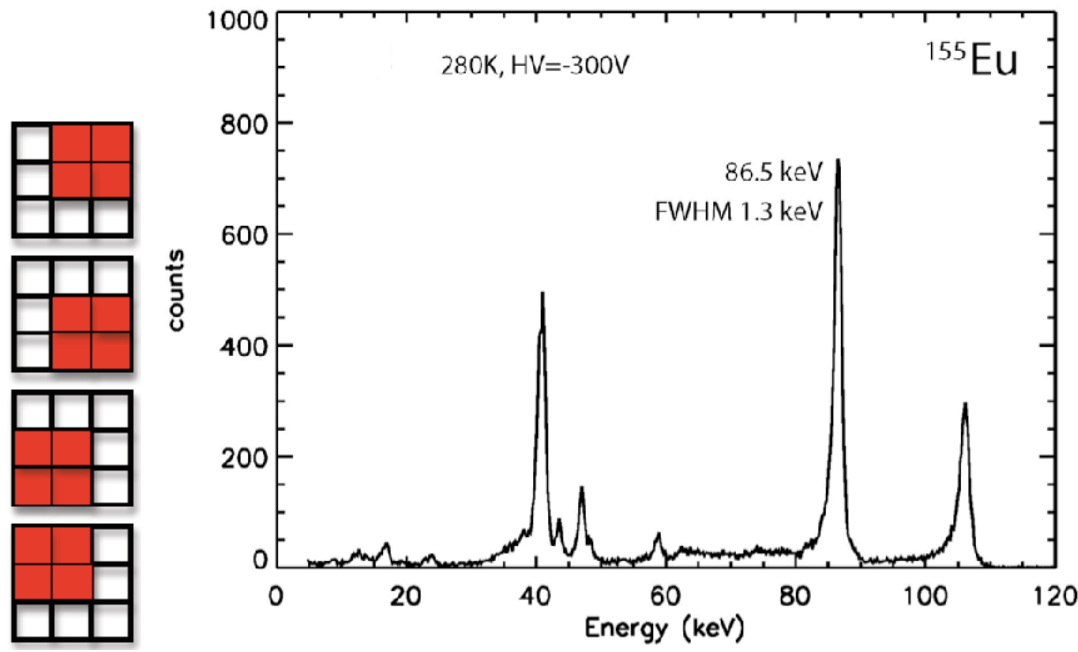

Figure 6: Left - Pixel dependent charge sharing patterns for a 9-pixel readout for up to 4-triggered pixels. Each column represents 1,2,3 and 4 triggered pixels respectively. Right $-{ }^{155} \mathrm{Eu}$ spectrum from CZT detector at $7 \mathrm{C}$ and $-300 \mathrm{~V}$ bias voltage for a $3 \times 3$ pixel region. All kind of events has been taken into account including charge sharing between several pixels.

\section{SUMMARY}

We evaluated six CdZnTe hybrids as part of the NuSTAR engineering model development. A detailed evaluation of these hybrids is still ongoing. However, some early results obtained from the characterization of these hybrids are summarized below.

1. The characterization of the hybrids demonstrates that the requirements for the NuSTAR mission will be met or exceeded for the CZT detectors.

2. Our custom ASICs bonded with the CZT has demonstrated low electronic noise, consistent with FWHM resolution of $<300 \mathrm{eV}$.

3. Most of our detectors show a relatively uniform distribution of counts over area, but all indicate some nonuniformity in pixels sizes due to transverse electric field components. Some detectors also show significant non-uniformities due to material variations.

4. The evaluation of spectral performance of the hybrids indicates that they meet NuSTAR requirement of $\leq 1.6$ $\mathrm{keV}$ FWHM for the energy resolution. Our detailed study indicates that to achieve ultimate spectral performance, we may need to correct for pixel-by-pixel detector gain variation and 13 different event topologies for each pixel. We are in the process of developing an efficient way to obtain these parameters and apply them to X-ray spectrum.

5. We will be testing and characterizing more than 50 CZT hybrids to find best 8 flight hybrids for NuSTAR. Thus future work will establish requisite corrections and calibrations for these hybrids to optimize them for best possible science output. 


\section{ACKNOWLEDGEMENTS}

This work was supported by the NASA APRA program under grant NAG and under the NASA NuSTAR contract, NNG06WC20G.

\section{REFERENCE}

[1] Barrett, H. H., Eskin, J. D. and Barber, H. B., "Charge Transport in Arrays of Semiconductor Gamma-Ray Detectors", Physical Review Letters, 75, 156-159 (1995).

[2] Rossi, L., Fischer P., Rohe T. and Wermes, N., "Pixel Detectors-From Fundamentals to Applications", Springer Publications, (2006).

[3] Harrison, F. H., et al., "Development of the High-Energy Focusing Telescope (HEFT) Balloon Experiment", Proc. SPIE Vol. 4012, 693-699, (2000)

[4] Koglin, J. E., et al., "NuSTAR Hard X-ray Optics", Proc. SPIE Vol. 5900, 266-275, (2005)

[5] Koglin, J. E., et al., "NuSTAR Hard X-ray Optics Design and Performance", Proc. SPIE, to appear in this volume. 